\title{
morphMan: Automated manipulation of vascular geometries
}

\author{
Henrik A. Kjeldsberg ${ }^{1}$, Aslak W. Bergersen ${ }^{1}$, and Kristian \\ Valen-Sendstad $^{1}$
}

1 Department of Computational Physiology, Simula Research Laboratory

DOI: $10.21105 /$ joss. 01065

\section{Software}

- Review ¿

- Repository $\boldsymbol{C}^{\boldsymbol{1}}$

- Archive ct

Submitted: 25 October 2018

Published: 20 March 2019

\section{License}

Authors of papers retain copyright and release the work under a Creative Commons Attribution 4.0 International License (CC-BY).

\section{Summary}

Cardiovascular diseases are overwhelming the healthcare systems, and the costs are anticipated to increase in the years to come (Murray \& Lopez, 1997), not to the mention the personal tragedy for those affected (Gage, Cardinalli, \& Owens, 1996). Systemic risk factors are well known to correlate with cardiovascular diseases in general, but, for instance, arterial plaques and brain aneurysms are focalized, highlighting the role of local hemodynamics. Furthermore, blood-flow induced wall shear stress (WSS) is known to contribute to vessel wall adaption and remodeling (A. M. Malek, Alper, \& Izumo, 1999; Morbiducci et al., 2016), but is challenging to measure in-vivo. On the other hand, medical images are routinely available and have been extensively used in combination with computational fluid dynamics to study the initiation, progression, and outcome of vascular pathologies (Taylor \& Steinman, 2010).

We know that the morphological features of, for instance, the internal carotid artery is statistically associated with the presence of aneurysms (T. Ingebrigtsen et al., 2004; Schimansky, Patel, Rahal, Lauric, \& Malek, 2013). Therefore, understanding how the local hemodynamics change with morphology is of great interest and is typically investigated with parameterized idealized geometric models (Lauric et al., 2018), however at the cost of oversimplified results. To use realistic geometries we could instead correlate the shape and computed stresses based on hundreds of patient-specific models, but this is very labor intensive and error-prone (Berg et al., 2018; Valen-Sendstad et al., 2018).

Our goal was to take the best from both approaches and create a tool which could parametrize patient-specific geometries to mimic the natural variability of morphological features in the population. We here present a framework, morphMan, that allows for objective, reproducible, and automatic virtual manipulation of tubular structures here exemplified with application to the cerebrovasculature. 


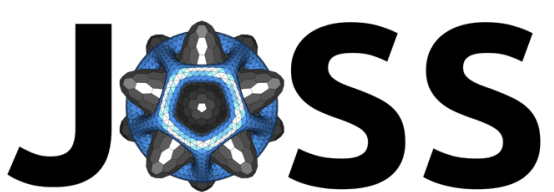

The Journal of Open Source Software

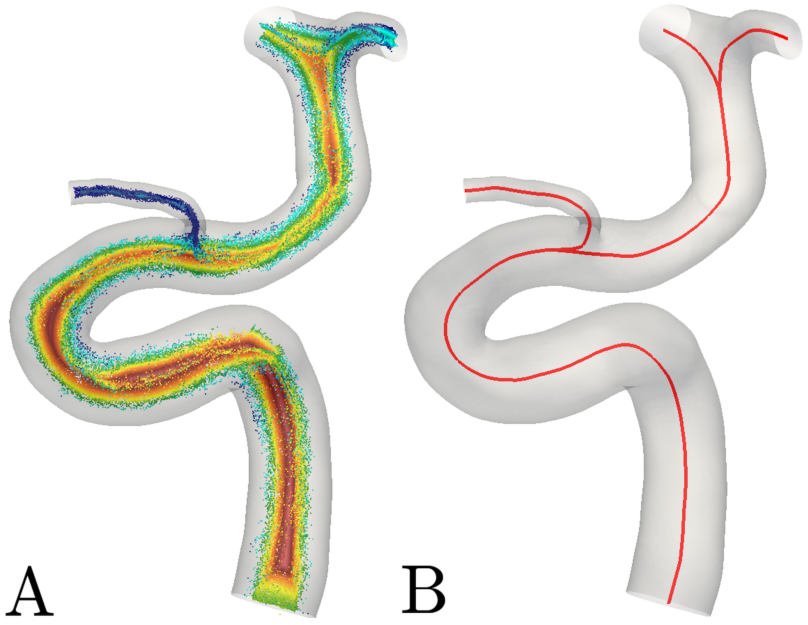

Figure 1: A visualization of the Voronoi diagram (left) and the centerline (right) of a surface.

In a surface, each cell is connected, and manipulating one will alter the surrounding geometry as well. Instead, we have based the algorithms on the centerlines and Voronoi diagram of the surface, see Figure 1. The point in the Voronoi diagram are not connected, and therefore easier to manipulate. As a result, only the region of interest is manipulated, and the rest of the geometry is left unchanged. Using the Voronoi diagram to alter the surface was first presented in (Piccinelli et al., 2011); moreover, a subset of the algorithms are presented in (A. W. Bergersen, 2016) and (Kjeldsberg, 2018).

In morphMan v0.2 you can alter cross-sectional area, bifurcation angles, overall curvature in a segment, and the shape of specific bends. For each category, there is a wide range of options, thus providing the users with many degrees of freedom for manipulating the geometries. Shown in Figure 2 is an example of rotating the branches in a bifurcation 'up' and 'down'.

The intended audience for morphMan are researchers, particularly within, but not limited to, computational biomechanics. For instance, by combining morphMan with a computational fluid mechanics solver, it can be used to objectively and reproducibly investigate how a specific geometric feature impacts the local hemodynamics. Following the tutorial, there are now prior knowledge needed, except for how to run a program from the terminal.

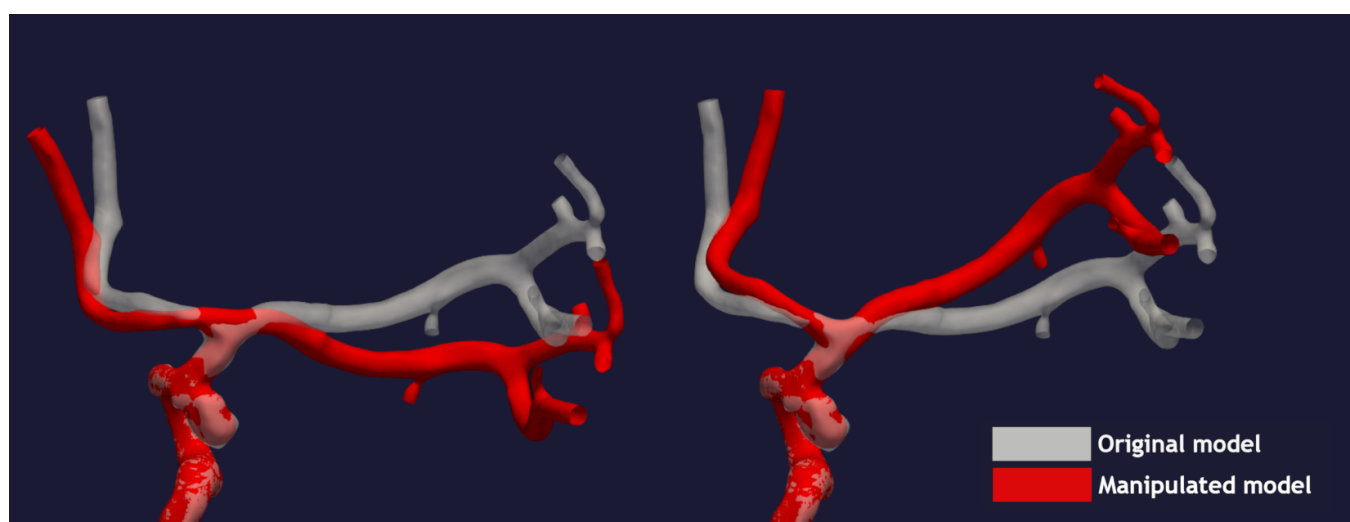

Figure 2: Output from morphMan for manipulating the bifurcation angles.

To summarize, morphMan is a general tool for automated manipulation of tubular objects, easily expandable for specialized manipulations. In the context of flow in the cardiovas-

Kjeldsberg et al., (2019). morphMan: Automated manipulation of vascular geometries. Journal of Open Source Software, 4(35), 1065. 2 https://doi.org/10.21105/joss.01065 
cular system, morphMan opens new lines of investigation for unraveling the coupling between morphology and the computed local hemodynamics.

\section{Acknowledgements}

We acknowledge Alban Souche for testing morphMan, and the two open-source projects vtk, and vmtk.

\section{References}

Berg, P., Vo B, S., Saalfeld, S., Janiga, G. á bor, Bergersen, A. W., Valen-Sendstad, K., Bruening, J., et al. (2018). Multiple aneurysms anatomy challenge 2018 (match): Phase i: Segmentation. Cardiovascular engineering and technology, 1-17. doi:10.1007/ s13239-018-00376-0

Bergersen, A. W. (2016). Investigating the link between patient-specific morphology and hemodynamics: Implications for aneurism initiation? (Master's thesis).

Gage, B. F., Cardinalli, A. B., \& Owens, D. K. (1996). The effect of stroke and stroke prophylaxis with aspirin or warfarin on quality of life. Archives of internal medicine, 156(16), 1829-1836. doi:10.1001/archinte.156.16.1829

Ingebrigtsen, T., Morgan, M. K., Faulder, K., Ingebrigtsen, L., Sparr, T., \& Schirmer, H. (2004). Bifurcation geometry and the presence of cerebral artery aneurysms. Journal of neurosurgery, 101(1), 108-113. doi:10.3171/jns.2004.101.1.0108

Kjeldsberg, H. A. (2018). Investigating the interaction between morphology of the anterior bend and aneurysm initiation (Master's thesis).

Lauric, A., Greim-Kuczewski, K., Antonov, A., Dardik, G., Magida, J. K., Hippelheuser, J. E., Kono, K., et al. (2018). Proximal parent vessel tapering is associated with aneurysm at the middle cerebral artery bifurcation. Neurosurgery. doi:10.1093/neuros/nyy 152

Malek, A. M., Alper, S., \& Izumo, S. (1999). Hemodynamic Shear Stress and Its Role in Atherosclerosis. JAMA, 282(21), 2035-2042. doi:10.1001/jama.282.21.2035

Morbiducci, U., Kok, A. M., Kwak, B. R., Stone, P. H., Steinman, D. A., Wentzel, J. J., \& others. (2016). Atherosclerosis at arterial bifurcations: Evidence for the role of haemodynamics and geometry. Thrombosis and Haemostasis, 115(3), 484-492. doi:10. 1160/th15-07-0597

Murray, C. J., \& Lopez, A. D. (1997). Alternative projections of mortality and disability by cause 1990-2020: Global burden of disease study. The Lancet, 349(9064), 1498-1504. doi:10.1016/s0140-6736(96)07492-2

Piccinelli, M., Bacigaluppi, S., Boccardi, E., Ene-Iordache, B., Remuzzi, A., Veneziani, A., \& Antiga, L. (2011). Geometry of the internal carotid artery and recurrent patterns in location, orientation, and rupture status of lateral aneurysms: an image-based computational study. Neurosurgery, 68(5), 1270-85; discussion 1285. doi:10.1227/NEU. 0b013e31820b5242

Schimansky, S., Patel, S., Rahal, J., Lauric, A., \& Malek, A. M. (2013). Extradural internal carotid artery caliber dysregulation is associated with cerebral aneurysms. Stroke, 44(12), 3561-3564. doi:10.1161/strokeaha.113.001762

Taylor, C. A., \& Steinman, D. A. (2010). Image-based modeling of blood flow and vessel wall dynamics: Applications, methods and future directions. Annals of biomedical engineering, 38(3), 1188-1203. doi:10.1007/s10439-010-9901-0 
Valen-Sendstad, K., Bergersen, A. W., Shimogonya, Y., Goubergrits, L., Bruening, J., Pallares, J., Cito, S., et al. (2018). Real-world variability in the prediction of intracranial aneurysm wall shear stress: The 2015 international aneurysm cfd challenge. Cardiovascular engineering and technology, 1-21. doi:10.1007/s13239-018-00374-2 